\title{
RESULTS FROM A SWEPT-FREQUENCY IONOSPHERIC IMPEDANCE PROBE
}

\author{
E. K. MILI.ER \\ Lawrence Livermore Laboratory, University of California, Livermore, California, U.S.A. \\ and \\ H. F. SCHULTE, JR. \\ College of Engineering, University of Michigan, Ann Arbor, Michigan, U.S.A.
}

(Received 17 December 1973)

\begin{abstract}
Experimental results are presented for the impedance of a rocket-borne dipole antenna immersed in the ionospheric plasma. The dependence of several interesting impedance artifacts upon the antenna position relative to the Earth's magnetic field and rocket motion through the ionospheric plasma are shown. Possible evidence for plasma compressibility is provided by an impedance discontinuity occurring consistently at approximately twice the electron cyclotron frequency and a frequency shifted cyclotron-resonance impedance minimum.
\end{abstract}

\section{INTRODUCTION}

The use of rocket and satellite-borne RF (radio frequency) antennas as diagnostic probes in the ionosphere is well established, having been discussed as early as 1952 by Jackson and, among others, by Heikkila et al. (1967) and, more recently, Bishop and Baker (1972). On a less firm basis, however, is the interpretation and reduction of the probe data thereby obtained to ionospheric parameters such as electron density and collision frequency. The reasons for this are twofold: first, a lack of sufficiently comprehensive and systematic experimental data for the determination of the significant physical processes involved; second, an inadequate theoretical capability for predicting the behavior of an antenna in the ionospheric plasma.

The experiment to be discussed here was undertaken in connection with a parallel theoretical effort to determine the operating characteristics of an antenna carried aboard a rocket in the ionosphere, as well as to demonstrate, by a comparison of various theoretical models with the experimental results, the plasma properties to which the antenna is most sensitive. Since a theoretical formulation of an antenna in the ionosphere that realistically takes into account all the likely significant features, such as the sheath, plasma anisotropy, plasma compressibility, etc., is hopelessly complicated, one must be satisfied to perform an analysis that is, at best, approximate. Even with a restricted theoretical approach, however, one can possibly ascertain the cause of some of the experimentally observed features of the antenna impedance. Then, logical explanations concerning some of the measured results that the theory does not explain can be proposed.

Most antenna payloads used for ionospheric experimentation in the past have used one or several fixed operating frequencies. The reason for using fixed frequencies was, partly, that they simplify the experimental apparatus; also, under the proper operating conditions (e.g. operating frequency $f>$ electron plasma frequency $f_{p}$ ), one may possibly obtain reasonably accurate values for $f_{p}$ from the measurements. It is clear, however, that, if a more complete picture of the antenna-plasma interaction is to be obtained, one should use a continuous frequency sweep over a band that includes the particular frequencies of interest such as $f_{p}$, the electron cyclotron frequency $f_{h}$, and the upper hybrid frequency $f_{t}=$ $\sqrt{f_{p}^{2}+f_{h}^{2}}$. For this reason, the rocket for which some results are to be presented below carried a swept-frequency antenna experiment. This paper presents the advantages of this approach both for the antenna-impedance measurement and for some related experiments 
which will be described in the next section. In the third section, some of the experimental results are given along with some theoretical considerations related to interpreting rhe experimental data.

\section{THE EXPERIMENT}

Historically, many methods of varying degrees of complexity have been used in a search for a simple and reliable method of obtaining electron-density profiles in the ionosphere. When an ionosonde cannot be used because of ionospheric disturbances of $F 2$ peak height limitations, direct probes with rocket vehicles are generally made. Techniques that are sensitive to vehicle sheath or wake effects should be avoided if possible, a requirement leading naturally to the $R F$ probe, which has the possibility of sensing the ambient electron plasma frequency outside the sheaths of both the vehicle and probe itself. Heikkila $e t$ al. (1967) describe several $R F$ probe techniques, three of which (antenna impedance, resonance relaxation, and transmission experiments) have been selected for further study in our program.

A comprehensive description of the experimental details has been given elsewhere (Miller and Schulte, 1970). Here we limit our discussion to results obtained from measuring the absolute impedance of a 4.58-m half-length, $2 \mathrm{~cm}$ dia, balanced electric dipole. Data was obtained over a $0 \cdot 8-10 \mathrm{MHz}$ frequency range by exciting one half of the dipole with a swept-frequency voltage of approx $4 \mathrm{~V}$ r.m.s. in two overlapping bands. Each complete sweep required slightly over $4 \mathrm{sec}$ with 42 upleg and 52 downleg sweeps being obtained as the Aerobee rocket (NASA 4.207) was flown to an altitude of $292 \mathrm{~km}$ on 8 August 1967 at White Sands, New Mexico.

\section{EXPERIMENTAL RESULTS}

A typical result for the half-dipole impedance over one complete frequency sweep is shown in Fig. 1. Also included on this graph are three computed curves. Two are for the

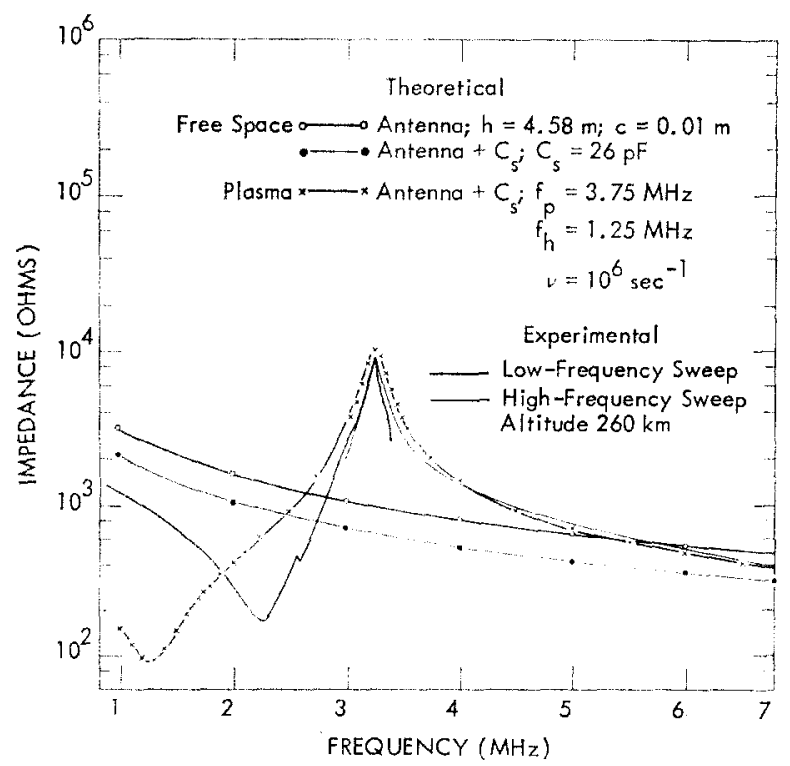

FIG. 1. EXPERIMENTAL HALF-DIPOLE IMPEDANCE PLOTTED VS FREQUENCY, TOGETHER WITH THE THEORETICAL IMPEDANCE FROM BALMAIN'S FORMULA FOR THE PLASMA MEDIUM, INCLUDING THE SHUNT CAPACITANCE, AND THE FREESPACE IMPEDANCE WITH AND WITHOUT THE SHUNT CAPACITANCE. 
antenna in free space, showing the effect of the stray shunt capacitance $C_{s}$ between the internal antenna mount and the rocket. The computed impedance curve for the plasmaimmersed antenna, which includes $C_{s}$, uses Balmain's (1964) quasistatic formula for the parameter values indicated on the figure and the antenna parallel to the static magnetic field. The values for $f_{p}$ and $v$ were chosen to obtain the apparent best-fit theoretical curve to the experimental results, although no attempt was made to accomplish this by a statistical procedure.

The collision frequency value of $10^{6} \mathrm{sec}^{-1}$ required for the theoretical impedance curve to fit the experimental data is unrealistically high for an altitude of $260 \mathrm{~km}$. A possible explanation for this may be that proposed by Balmain et al. (1967), who found from a quasistatic analysis of a spherical antenna in a cold magnetoplasma that a radially variablesheath electron density produced an effect on the impedance similar to that caused by electron collisions in the absence of a sheath. Balmain et al. found that the high electroncollision frequency required to match the theoretical and experimental data was thus reduced to a more realistic value by including a sheath, a finding that appears to be applicable in our case, also.

It may be noticed that the antenna impedance peaks considerably below the upper hybrid frequency of $3.93 \mathrm{MHz}$ due to the effect of $C_{s}$. It may furthermore be seen that the experimental impedance results approach the free-space theoretical curve, which includes the effect of $C_{s}$, from above for frequencies above $f_{t}$.

For frequencies below $f_{h}$, the measured impedance appears to be approaching a value less than that predicted for a free-space, apparently reflecting the effect of a sheath capacitance. We can write the input impedance to the antenna in the form

$$
Z_{I N}=Z_{S} \tilde{Z}_{A} /\left(Z_{S}+\tilde{Z}_{A}\right)
$$

where $Z_{S}$ and $\tilde{Z}_{\mathcal{A}}$ are the shunt capacitance and antenna-sheath combination impedances. Furthermore, from Balmain (1966) we have, assuming a vacuum sheath for a model,

$$
\tilde{Z}_{A}=Z_{R, \text { freespace }}-Z_{S, \text { freespace }}+Z_{S, \text { plasma }}
$$

with $R$ and $S$ denoting the antenna and sheath radii respectively and $Z$ the impedance of a dipole of the corresponding radius in free-space or the plasma. Upon solving for $\tilde{Z}_{A}$ from (1) we find

$$
\tilde{Z}_{A}=Z_{S} Z_{I N} /\left(Z_{S}-Z_{I N}\right)=Z_{S H}+Z_{A}
$$

where $Z_{S H}$ is the sheath impedance $\left(Z_{R}\right.$. freespace $\left.-Z_{S \text {, freespace }}\right)$ and $Z_{A}$ is the antenna impedance $\left(Z_{S, \text { plasma }}\right)$. Finally then, since the sheath impedance is predominantly capacitive,

$$
Z_{S H}=Z_{S} Z_{I N} /\left(Z_{S}-Z_{I N}\right)-Z_{A} \sim \frac{1}{j \omega C_{S H}}
$$

from which we can find $C_{S H}$ given $Z_{A}$. Since we do not know the sheath radius, and since in addition $Z_{A}$ is not strongly dependent upon the radius, we use $Z_{A} \sim Z_{R}$, plasma. If we denote by $\widetilde{Z}_{A}$ the antenna impedance results computed from Balmain (1966) which include the effect of the shunting capacitance but not the sheath as shown on Fig. 1, we finally have

$$
C_{S H} \sim\left|\frac{\left(Z_{S}-Z_{I N}\right)\left(Z_{S}-\widetilde{\widetilde{Z}}_{A}\right)}{\omega Z_{\mathrm{S}}^{2}\left(Z_{I N}-\widetilde{\widetilde{Z}}_{A}\right)}\right|
$$


Some representative results for $C_{S I I}$ as determined from Equation (5) using the measured values for $Z_{I N}$ and calculated results for $\widetilde{Z}_{A}$ at a frequency of $1 \mathrm{MHz}$ follow below in Table 1. Note that $Z_{I N}$ has been assumed to be purely capacitive in using Equation (5). This is reasonable at this frequency, and necessary as well, since our measurement provides the absolute impedance value only. Also included is an estimation of the equivalent vacuum sheath thickness computed from $Z_{S \text {, freespace }} \sim Z_{R \text {, freespace }}-Z_{S H}$ using the quasistatic formula given by Balmain (1966), to calculate an antenna impedance value.

TABLE 1

\begin{tabular}{ccccc}
\hline $\begin{array}{c}\text { Height } \\
(\mathrm{km})\end{array}$ & $\begin{array}{c}Z_{W N} \\
\text { (ohms) }\end{array}$ & $\begin{array}{c}\widetilde{Z}_{A} \\
\text { (ohms) }\end{array}$ & $\begin{array}{c}C_{S H} \\
(\mathrm{pfd})\end{array}$ & $\begin{array}{c}S_{S A} \\
(\mathrm{~cm})\end{array}$ \\
\hline 105 & 2120 & 1250 & 95 & $15 \cdot 0$ \\
145 & 1575 & 650 & 114 & $9 \cdot 3$ \\
182 & 1300 & 325 & 122 & $8 \cdot 1$ \\
235 & 1260 & 210 & 117 & $8 \cdot 8$ \\
260 & 1260 & 160 & 111 & $9 \cdot 9$ \\
282 & 1180 & 140 & 121 & $8 \cdot 2$ \\
\hline
\end{tabular}

A slight decrease in the computed sheath capacitance with decreasing height may be observed. This results in an increased sheath radius due to the decreased election density, and consequently a larger value for the Debye length at the lower altitudes.

Further evidence is provided for the influence of a sheath on the impedance by the upward shift in frequency of the impedance minimum, predicted for the sheathless case to be located at $f_{h}$. This shift evidently occurs due to a series resonance between the capacitive sheath and the inductive plasma and is predicted both by Balmain's (1966) spherical antenna quasistatic analysis and Miller's (1967) treatment of the infinite cylindrical antenna in an incompressible, anisotropic plasma. Miller used a concentric free-space layer or vacuum sheath to approximate the actual inhomogeneous sheath. In addition, it is interesting to note that, for the infinite antenna, the plasma compressibility causes an upward shift of the impedance minimum for the anisotropic plasma when there is no sheath (Miller, 1968) and may thus represent an equivalent capacitance.

While we cannot, therefore, be sure of the relative influence these two factors exert in determining the impedance minimum, it is possible to estimate their combined capacitive effect. We may again employ Equation (5) to obtain an equivalent capacitance $C_{E Q}$, from the measured and computed impedance values. The results derived for $C_{E Q}$ are summarized in Table 2 below for the same altitudes employed in Table 1. Also included is a computed sheath thickness denoted by $S_{E Q}$ using the same procedure as described above and assuming $C_{E Q}$ is due entirely to a sheath effect.

TABLE 2

\begin{tabular}{llcccc}
\hline $\begin{array}{c}\text { Height } \\
(\mathrm{km})\end{array}$ & $\begin{array}{c}f_{m} \\
(\mathrm{MHz})\end{array}$ & $\begin{array}{c}\approx \\
Z_{I N} \\
(\mathrm{ohms})\end{array}$ & $\begin{array}{c}\widetilde{Z}_{A} \\
(\mathrm{ohms})\end{array}$ & $\begin{array}{c}C_{\boldsymbol{E Q}} \\
(\mathrm{pfd})\end{array}$ & $\begin{array}{c}S_{\boldsymbol{Z Z}} \\
(\mathrm{cm})\end{array}$ \\
\hline 105 & $1 \cdot 48$ & 1300 & 1570 & 159 & $5 \cdot 0$ \\
145 & $1 \cdot 64$ & 780 & 1040 & 155 & $5 \cdot 2$ \\
182 & $1 \cdot 8$ & 420 & 740 & 177 & $4 \cdot 2$ \\
235 & $2 \cdot 2$ & 165 & 700 & 133 & $6 \cdot 8$ \\
260 & $2 \cdot 26$ & 170 & 620 & 145 & $5 \cdot 8$ \\
282 & $2 \cdot 28$ & 200 & 480 & 189 & $3 \cdot 8$ \\
\hline
\end{tabular}


In deriving the above $C_{E Q}$ results we have again had to make an assumption regarding the phase of $Z_{I N}$. Because the calculation is performed at the frequency of an impedance minimum, we assume $Z_{I N}$ here to be purely real.

It is intriguing to note upon comparing the results of Tables 1 and 2 that $C_{E Q}>C_{S H}$ (and consequently $S_{E Q}<S_{S H}$ ) in every case. This seems to imply an 'excess' capacitance at the impedance minimum compared with that obtained from the $1 \mathrm{MHz}$ impedance data. If the sheath is entirely responsible for the capacitive effect, its capacitance defined above as $Z_{S H}=Z_{A, \text { freespace }}-Z_{B \text {, freespace }} \propto\left(\omega C_{S H}\right)^{-1}$ would not be expected to vary with frequency. One other possible source for the increased capacitance at $f_{m}$ is the plasma compressibility, whose effect might indeed be frequency dependent. The data cannot be regarded as conclusive in this regard. Since we are unable to provide an estimate of the various errors involved, we cannot say whether the difference between $C_{E Q}$ and $C_{S H}$ is significant. It is interesting to note, however, that Nakatani and Kuehl (1973) find from a kinetic theory formulation that the compressibility effects, negligible below $f_{h}$ so that Balmain's (1964) quasistatic theory is adequate there, do exhibit significant effects near harmonics of $f_{h}$. Our data would seen to be consistent with this finding.

A further demonstration of the possible influence of the plasma compressibility is provided by the impedance discontinuity, or dip, that occurs at approx $2 f_{h}$ on Fig. 1 . This feature consistently occurred on the impedance records throughout the flight, as well as being manifested by significant responses on both the relaxation resonance and transmission experiment channels. A resonance at harmonics of the cyclotron frequency was observed by the Alouette and Explorer satellites, as well as at about $2 f_{h}$ on another rocket impedance experiment (Melzner and Robben, 1970). These responses have been attributed to zeroes of the dispersion equation by Dougherty and Monaghan (1966); but, in the case of the antenna impedance, Johnston (1968) suggested that the use of a tensor rather than a scalar pressure for the compressible, anisotropic plasma would result in an impedance excursion at $2 f_{h}$. As mentioned above, it also appears consistent with the kinetic theory results of Nakatani and Kuehl.

The experimental-theoretical impedance comparison shown on Fig. 1 is typical of the agreement obtained between measured data and calculated best-fit values for the impedance throughout the flight. It appears that one may confidently derive electron densities from the measured impedance results by this approach, which makes use of Balmain's theory. It is unnecessary to go through the labor of fitting the entire curve, however, to obtain $f_{p}$, which may instead be more easily found from the impedance magnitude at a fixed frequency above $f_{h}$ where the influence of $v$ is relatively unimportant. Alternatively, the frequency of the impedance peak below $f_{t}$ can also be used for this purpose, since here again the frequency at which this occurs is insensitive to $v$ (for $v<\omega_{y}$ ). Altitude profiles of the electron density have been derived from the impedance data for the flight, and found to compare favorably with a simultaneous ground-based ionosonde record as well as with an on-board Langmuir probe. Some preliminary results of this analysis have been given by the authors (Schulte et al., 1968); a more complete presentation of the electron-density measurement using the impedance data concludes this discussion.

For the remainder of the paper we restrict our attention to the influence of the rocket rotation on the antenna impedance. The results presented below were derived from machine-plotted curves of the computer-processed impedance data. The data are given in two forms, the first set of graphs showing the frequencies at which various interesting impedance features occur, and the second showing the magnitudes of some of the same 
impedance features, both as functions of time. Since the rotation period of the rocket was about $20 \mathrm{sec}$, more than four complete frequency sweeps were obtained per period, thus providing sufficient resolution to demonstrate the impedance dependence on the magnetic field and rocket wake.

In Fig. 2 we show the frequency $f_{m}$ at which the impedance minimum, shifted upward

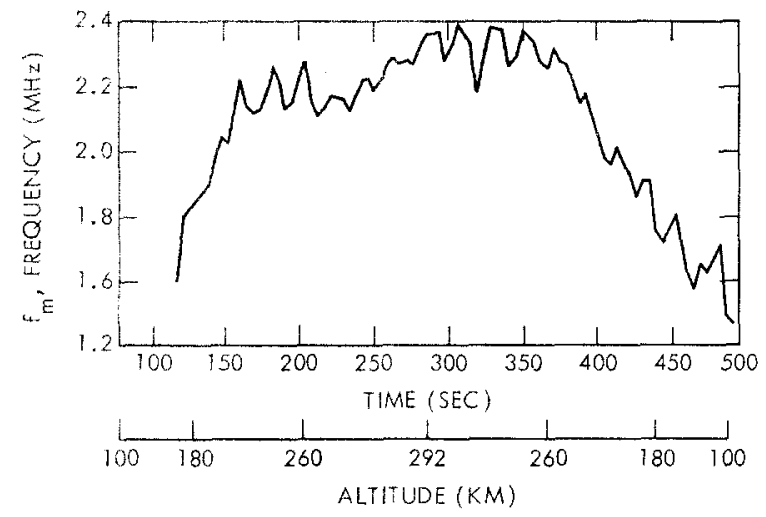

Fig. 2. The FRequenCY $f_{m}$ AT Which THE halF-DiPOLE IMPEDANCE MINIMUM $Z_{m}$ ASSOCIATED WITH THE ELECTRON CYCLOTRON FREQUENCY $f_{h}$ OCCURS AS A FUNCTION OF FLIGHT TIME.

from $f_{h}$, occurs. It should be recalled from the discussion above that this shift may be due to combination of sheath and compressibility effects. Figure 2 shows well-defined oscillatory pattern of $f_{m}$ in time that has a period corresponding to the rotation period of the rocket. This modulation of $f_{m}$ is strongly correlated with the antenna position relative to the rocket wake, as may be seen by comparing Fig. 2 with Fig. 3, which shows the stream velocity tangential to the antenna. The velocity is taken to be positive when the stream

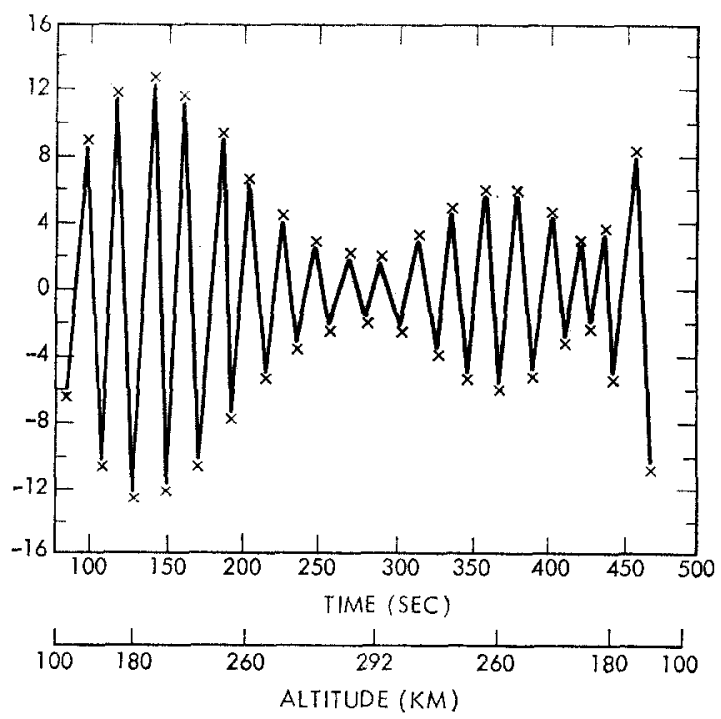

Fig. 3. The plasma-stream velocity tangential to the half-Dipole whose impedance WAS MEASURED, AS A FUNCTION OF FLIGHT TIME.

Positive velocity values indicate that the half-dipole points in the direction of rocket motion. 
impinges on the rocket, so that the antenna is then facing in the direction of rocket motion, while for negative velocities the antenna is located on the trailing side of the rocket and passing through its wake. The value of $f_{m}$ may be seen to be largest when the antenna is facing forward into the unperturbed (by the rocket) ambient ionospheric plasma. Since, as discussed above, the upward shift of $f_{m}$ from $f_{h}$ may be due to both sheath and compressibility effects, it is difficult to conclude at this point the more important of these two factors in determining $f_{m}$.

In Fig. 4 theoretical values of $f_{m}$ are shown for an infinite cylindrical antenna as functions

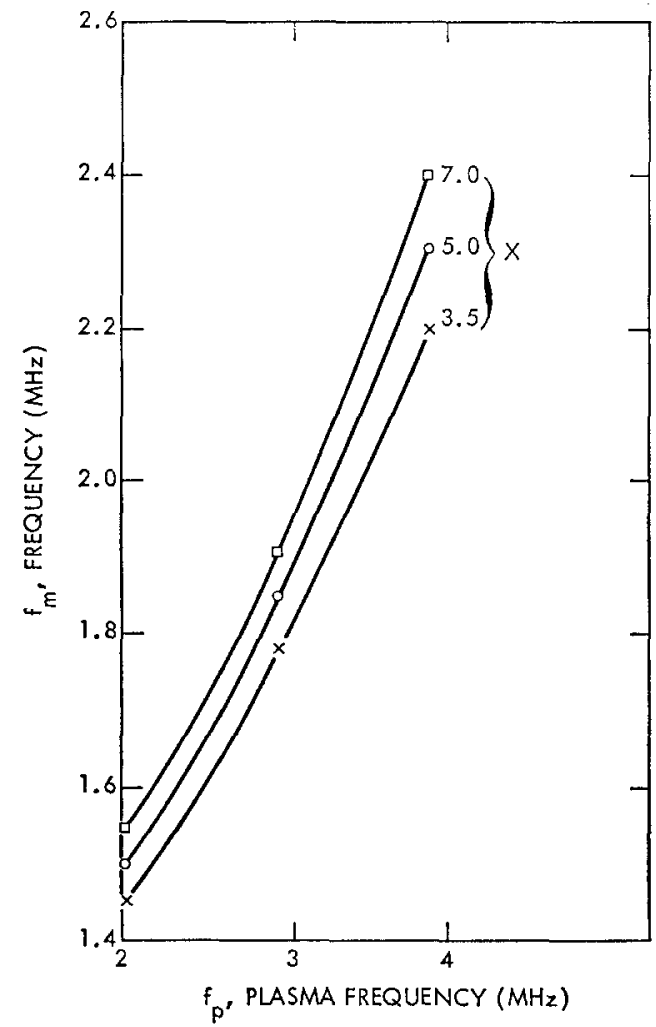

Fig. 4. Theoretical values for $f_{m}$ as a function of the Plasma frequency $f_{\eta}$, with The VACUUM SHEATH THICKNESS $X$ IN DEBYE LENGTHS AS A PARAMETER, FOR THE INFINITE CYLINDRICAL ANTENNA.

of $f_{p}$, with the thickness of the vacuum sheath a parameter. This vacuum sheath thickness $X$ is given in units of the Debye length $\left(D_{l}=V_{0} / \omega_{p} \sqrt{ } 3\right.$, where $V_{0}=$ r.m.s. electron velocity $)$ for an electron temperature $T$ of $1500^{\circ} \mathrm{K}$, with $f_{h}=1.25 \mathrm{MHz}$ and $v=10^{4} \mathrm{sec}^{-1}$. We see that the upward shift of $f_{m}$ is considerably more sensitive to increasing the value of $f_{p}$ than to the vacuum-sheath thickness, despite the fact that the physical sheath thickness for a fixed value of $X$ decreases with increasing $f_{p}$. This result indicates that $f_{p}$ is larger in front of the rocket than in its wake, a conclusion that is further substantiated by the impedance values measured above $f_{t}$ where the sheath influence is relatively unimportant and which will be further discussed below.

The frequency at which the impedance maximum associated with the upper hybrid frequency $f_{t}$ occurs, denoted by $f_{M}$, is shown in Fig. 5 . It should be recalled that $f_{M}$ is not 


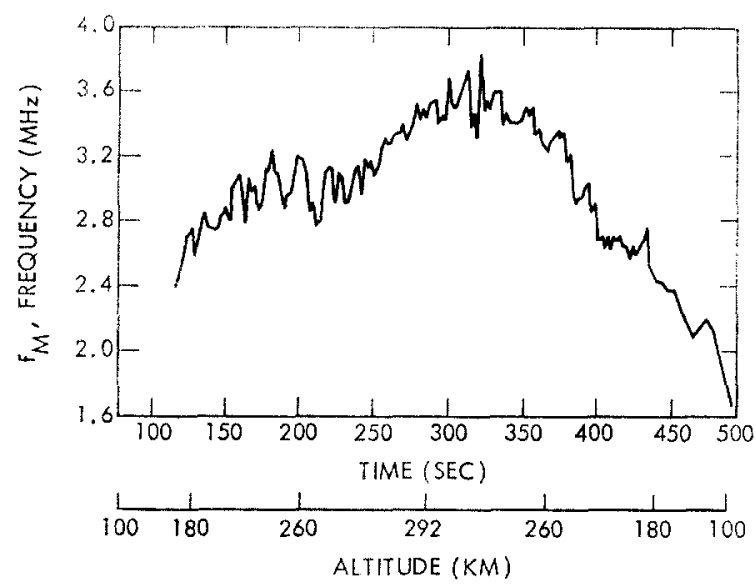

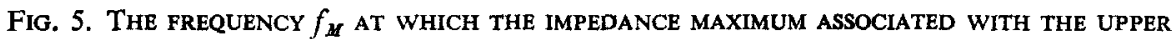
HYBRID FREQUENCY $f_{t}$ OCCURS, AS A FUNCTION OF FLIGHT TIME.

equal to $f_{t}$ because of the antenna shunt capacitance $C_{s}$. The oscillation of $f_{M}$ with time is seen to be about twice as fast as that exhibited by $f_{m}$ on Fig. 4 and is well-correlated with the tangential component of the ionospheric magnetic field at the antenna, the time variation of which is shown in Fig. 6. It appears that the value of $f_{M}$ is sensitive to the absolute magnitude of the tangential magnetic field at the antenna, and further that $f_{M}$ has maxima at extrema of the tangential magnetic field. This result agrees with Balmain's (1964) quasistatic theory of a cylindrical dipole antenna that shows the impedance to be a function of the absolute value of the magnetic field and the absolute magnitude of the angle between the antenna axis and the magnetic field.

The concluding graph of this series is a time plot of the two frequencies at which discontinuities or sudden changes in the impedance were consistently found throughout the flight. The lower of these two frequencies, which we denote as $f_{d 1}$, appears to be approx twice the election cyclotron frequency and is relatively insensitive to altitude, as may be

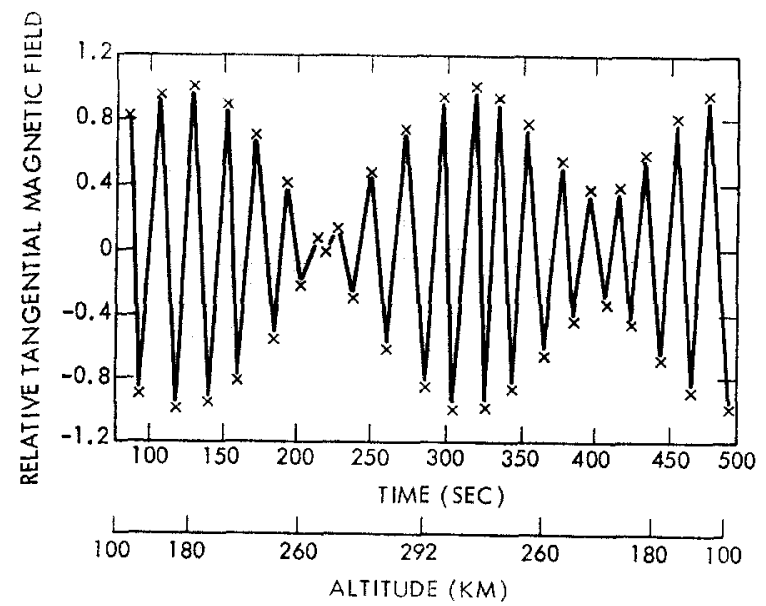

Fig. 6. The tangential Component of magnetic field at the dipole, as a function of FLIGHT TIME. 


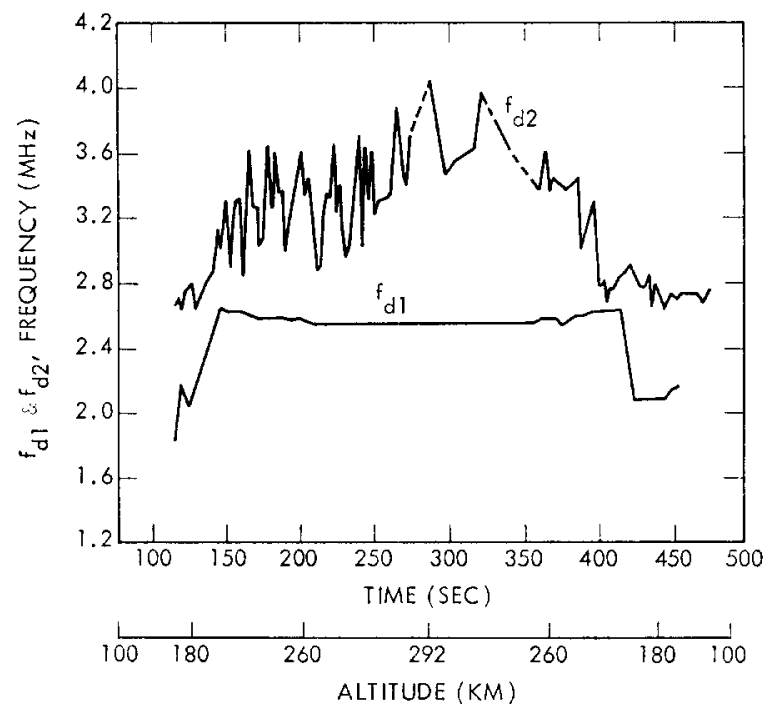

Fig. 7. A PLOT OF the TWO FREQUeNCIES AT WHiCh IMPEDANCE DISCONTINUITIES WERE OBSERVED CONSISTENTLY THROUGHOUT THE FLIGHT, AS FUNCTIONS OF FLIGHT TIME.

The frequency at approx twice $f_{h}$ is denoted by $f_{d 1}$, and that associated with $f_{p}$ is denoted by $f_{d 2}$.

seen on Fig. 7. The value of $f_{d 1}$ may be observed to be smallest over the middle portion of the flight, as would be expected, since the value of $f_{h}$ decreases with altitude. It is interesting to note that recognizable responses at this same frequency of about $2 f_{h}$ were consistently observed throughout the flight on the transmission and relaxation resonance experiment channels, also. The higher of the two frequencies, which we denote by $f_{d 2}$, is seen to be a much more variable function of time than $f_{d 1}$. (The dashed portion of the curve indicates regions where values for $f_{d 2}$ could not be obtained from the data, principally because of the masking effect of the downward-shifted $f_{t}$ peak.) A comparison of the $f_{d 2}$ results on Fig. 7 with the tangential magnetic field component shown in Fig. 6 reveals some degree of correlation between them. There is a considerably greater correlation between $f_{d 2}$ and the normal stream velocity at the antenna, however, as a comparison of Figs. 7 and 3 shows. An impedance discontinuity is predicted to occur at $f_{p}$ by Balmain's (1964) quasistatic analysis as well as by the infinite-antenna results (Miller, 1967). The correlation of $f_{d 2}$ with the stream velocity (or antenna location relative to the rocket wake) indicates that the discontinuity thus may be used with reasonable confidence to determine $f_{p}$, as well as taken to demonstrate the validity of the theoretical results. The impedance discontinuity has been previously used by Heikkila et al. (1967) to find $f_{p}$. We may remark that the $f_{d 2}$ results of Fig. 7 are consistent with the $f_{m}$ values of Fig. 2 in indicating that $f_{p}$ is larger in the unperturbed plasma in front of the rocket than in the wake.

The second series of graphs are time plots of the impedance magnitudes of various interesting impedance features. In Fig. 8 are shown the magnitudes of the impedance minimum $Z_{m}$ at $f_{m}$ and the impedance maximum $Z_{M}$ at $f_{M}$. We see that the $Z_{m}$ values are for the most part correlated with the velocity curve of Fig. 3 . They exhibit a 1-cycle variation per rocket rotation rather than the 2 -cycle variation that would result from a magnetic field influence. A further intriguing feature of the $Z_{m}$ plot of Fig. 8 is the fact that the maximum values occur at negative velocity maxima, i.e. or when the antenna is in the rocket wake. This property of the impedance is also predicted by Balmain's theory, in that 


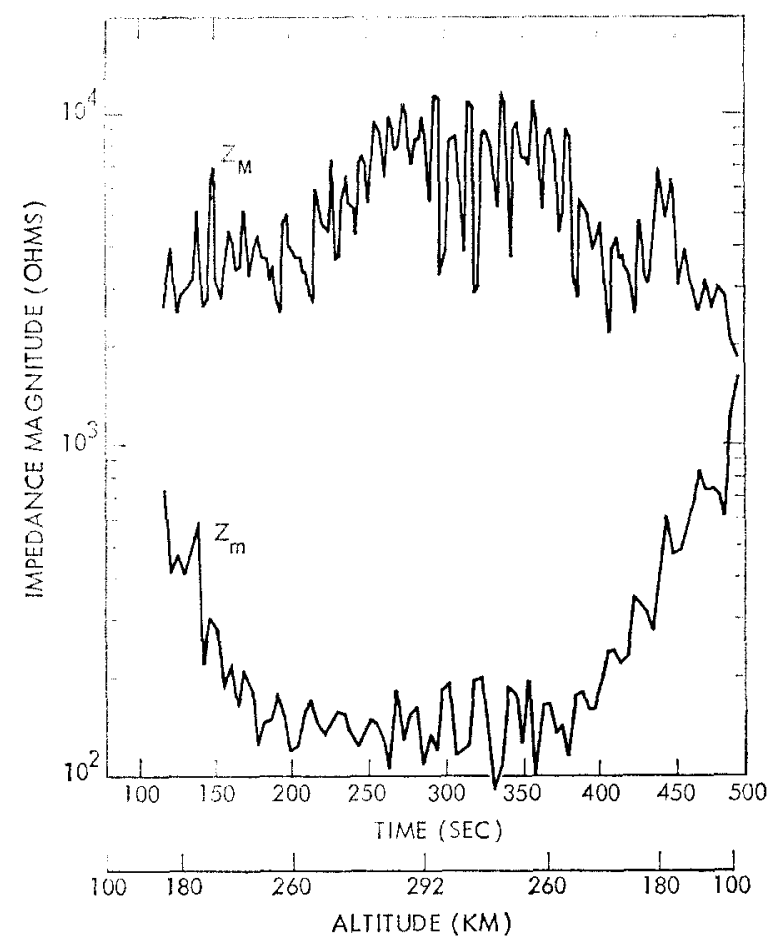

Fig. 8. The IMPedance minimum, $Z_{m}$, and maximum, $Z_{M}$, as fUNCtions of FLight time.

the impedance minimum at $f_{h}$ (which corresponds to our upward-shifted $Z_{m}$ impedance minimum) decreases as $f_{p}$ increases. We thus find a third measured impedance feature that indicates the reasonable result that the electron density is decreased in the rocket wake, compared to that in the unperturbed regions in front of the rocket.

Turning our attention now to the impedance maxima $Z$, we find a correlation with both the tangential velocity and tangential magnetic field components. Contrary to the finding for $Z_{m}$, it may be seen that $Z_{M}$ has maxima for positive maxima in the tangential stream velocity at the antenna. In addition to this, there are smaller maxima in $Z_{M}$ interspersed between those associated with the velocity, where it appears that $Z_{M}$ is maximized when the tangential magnetic field at the antenna is zero. Both of these features of the $Z_{M}$ values are also consistent with the predictions of Balmain's theory.

We find the impedance magnitude at the $f_{d 1}$ discontinuity, $Z_{d 1}$, as shown in Fig. 9 to be primarily correlated with the tangential velocity. The dependence upon the velocity is similar to that found in the $Z_{m}$ data above. This is to be expected, since the frequencies $f_{m}$ and $2 f_{h}$ are relatively close throughout the flight. A comparison of the $f_{d 1}$ curve of Fig. 7 with that of Fig. 11 further reveals that the sharp increases in $Z_{a 1}$ at the lower altitudes of the flight are correlated with similar abrupt decreases in the values of $f_{d i}$. It appears from this behavior in both $Z_{d 1}$ and $f_{d 1}$ that a significant change occurs in the phenomenon producing this impedance characteristic. The most obvious factor would be the altitude variation of the ionospheric plasma, since the transition region $Z_{d 1}$ and $f_{d 1}$ for both the upleg and downleg portions of the flight is between 190 and $200 \mathrm{~km}$, although it may be seen by referring to Fig. 5 that the larger tangential velocities also occur near the transition region of the $Z_{d 1}$ and $f_{d 1}$ behavior. 


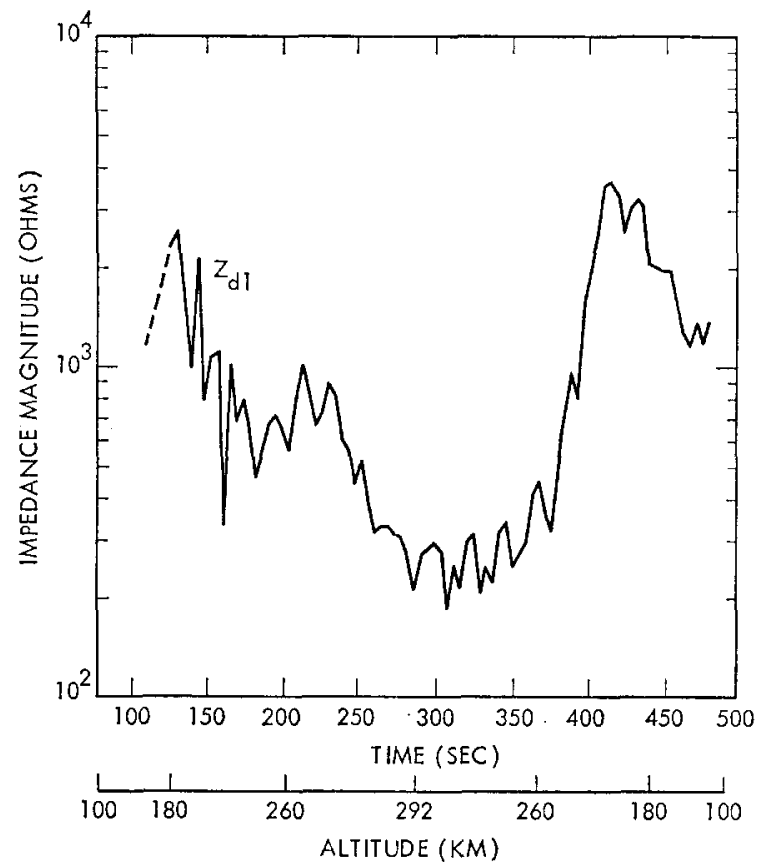

Fig. 9. The impedance value $Z_{d 1}$ OCCURring NEAR $2 f_{h}$, as a fUNCtion of Flight time.

As the final graph in this sequence, we present in Fig. 10 the impedance magnitude at a constant frequency of $5.25 \mathrm{MHz}$ plotted as a function of time during the flight. This frequency was chosen because it exceeded the highest value of $f_{t}$ encountered over the rocket trajectory, yet is close enough to $f_{t}$ to provide adequate sensitivity for the determination of $f_{p}$ from the impedance magnitude. A well-defined oscillatory behavior of the impedance magnitude is evident with a period of about $20 \mathrm{sec}$, the value of the rocket rotation

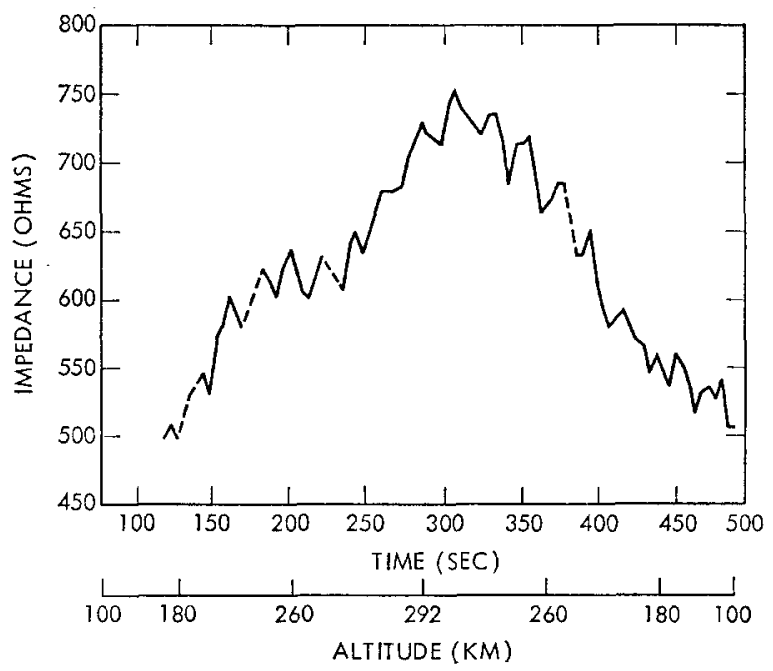

Fig. 10. The variations With Flight time of the impedance $Z_{5 \cdot 25}$ at $5 \cdot 25 \mathrm{MHz}$. These impedance data and the $f_{M}$ and $f_{d 2}$ values were used to obtain the electron-density results shown in Fig. 13. 
period. A comparison of Fig. 10 with Fig. 5 shows that the impedance minima occut at negative maxima of the tangential stream velocity at the antenna, i.e. when the antenna is located in the rocket wake. This finding provides additional evidence for a decreased electron density in the rocket wake, since theory predicts that above $f_{\imath}$ the impedance increases with increasing values of $f_{p}$. The general trend of the $5.25 \mathrm{MHz}$ impedance values to increase with altitude also demonstrates the increase in antenna impedance above $f$, which is caused by an increase in the electron density.

To conclude our discussion, we present in Fig. 11 a comparison of electron-density

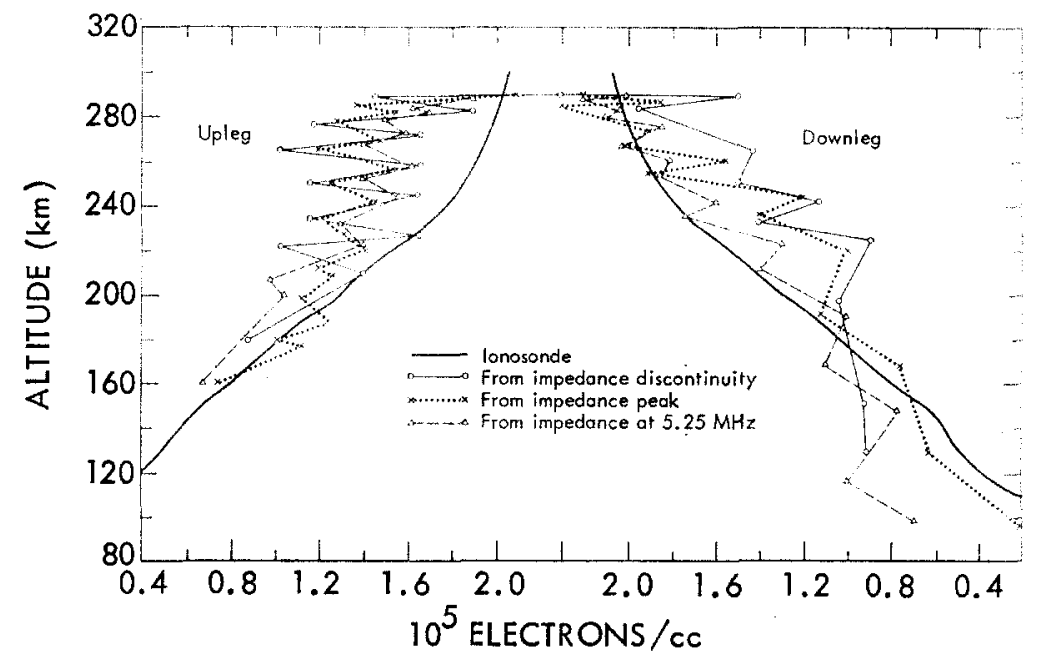

Fig. 11. A COMPARISON OF THE ELECTRON-DENSITY PROFILE MEASURED DURING THE FLIGHT. The heavy solid line is from ionosonde data; $\times$ points are from $f_{M} ; \bigcirc$ points are from $f_{a q}$; $\triangle$ points are from $Z_{5 \cdot 25}$.

values derived from the present experiment with independent data obtained from a groundbased ionosonde. The upleg and downleg portions of the flight are shown separately for clarity. Results are presented for three methods of deriving electron density from the measured antenna impedance: (1) the location in frequency of the peak impedance associated with the upper hybrid frequency; (2) the location in frequency of the impedance discontinuity associated with the electron plasma frequency; and (3) the impedance magnitude at $5.25 \mathrm{MHz}$. The former two methods use the frequency at which a particular impedance feature occurs and, insofar as the frequency value itself is concerned, should be accurate to within 1 per cent, since crystal-controlled markers are used to calibrate the frequency sweeps. The latter method, however, depends upon the ratio of the voltage and current at $5.25 \mathrm{MHz}$, as well as requiring the effect of a shunt capacitance upon the absolute impedance value to be accounted for, and so may provide a less accurate measure of electron density. We estimate the electron-density values overall to be experimentally accurate to \pm 20 per cent (Miller and Schulte, 1970).

The data points presented in Fig. 11 represent only the minimum and maximum values for electron number as obtained from plots of the kind shown in Fig. 10 and are connected by straight lines to improve their legibility. 
Agreement between the present results and the ionosonde curve is not too good for the upleg flight segment, but is considerably better on the downleg side. The impedancederived results exhibit fairly good consistency in their respective indications of electrondensity extrema as a function of altitude. The best overall correlation with the ionosonde data and our impedance results is found in the values derived from the frequency of the impedance peak. Except for the lower altitudes, the $5.25 \mathrm{MHz}$ results are also in good agreement with the ionosonde curve. Thus, in agreement with Heikkila et al. (1967) and Bishop and Baker (1972), we conclude that the impedance measurement provides a workable method for determining electron density.

\section{SUMMARY AND CONCLUSIONS}

The general findings of the experimental results presented above may be summarized as below.

(1) The measured impedance data, apart from a consistent discontinuity at $\sim 2 f_{h}$, and possibly the impedance of $f_{\min }$ are in accord with Balmain's (1967) quasistatic theory both as to dependence upon $f_{D}$ and $f_{h}$ and antenna orientation with respect to the magnetic field.

(2) Impedance values below $f_{h}$ (at $1 \mathrm{MHz}$ ) and at the upward shifted minimum associated with $f_{h}$, indicate a series capacitance, possibly due to a sheath, $\sim 100 \mathrm{pfd}$.

(3) The impedance magnitude above $f_{t}$ provides a reliable method for determining the electron density as does a consistently occurring discontinuity at $\sim f_{p}$.

The principal variations from quasistatic theory occur in impedance discontinuity observed at about $2 f_{h}$ and possibly also the upward shifted impedance minimum associated with $f_{h}$. The latter result could be explained as being due to the separate or combined influence of plasma compressibility or a sheath, based on the infinite antenna theory, and also qualitatively agrees with the kinetic theory results of Nakatani and Kuehl (1973).

In conclusion, the experimental values that have been obtained for the impedance of a rocket-borne antenna in the ionosphere demonstrate the potential value of an impedance probe for ionospheric diagnostics, its sensitivity to respond to small changes in the ambient plasma, and the value of a swept-frequency experiment for providing exceptionally useful data for comparison with theoretical predictions.

Acknowledgements-Portions of this work were performed under the auspices of the U.S. Atomic Energy Commission. The assistance of Mr. James Kuiper of the University of Michigan High Altitude Laboratory in obtaining the experimental data presented here is gratefully acknowledged.

\section{REFERENCES}

Balmain, K. G. (1964). The impedance of a short dipole antenna in a magnetoplasma. IEEE Trans. Antennas Propagation AP-12, 605.

Balmain, K. G. (1965). Impedance of a short dipole in a compressible plasma. Radio Sci. 69D, 559.

BALMAIN, K. G. (1966). Impedance of a spherical probe in a magnetoplasma. IEEE Trans. Antennas Propagation AP-14, 402.

Balmain, K. G., OKsiUTiK, G. and Fejer, J. A. (1967). RF probe admittance in the ionosphere: comparison of theory and experiment. Presented at Spring URSI Meeting, Ottawa, Ontario, Canada.

Bishop, R. H. and BAKER, K. D. (1972). Electron temperature and density determination from $R F$ impedance probe measurements in the lower ionosphere. Planet. Space Sci. 20, 997.

Dovgherty, J. P. and Monaghan, J. J. (1966). Theory of resonances observed in ionograms taken by sounders above the ionosphere. Proc. R. Soc. A289, 214.

Heikkila, W. J., Fejer, J. A., Hughill, J. and Calvert, W. (1967). Comparison of ionospheric probe techniques. Presented at VIIth Int. Space Sci. Symposium, Vienna.

JACKSON, J. E. (1952). Rocket-borne instrumentation for ionosphere propagation experiments. Upper Atmosphere Research Report No. VIII, Naval Research Laboratory, Washington, D.C.

Johnston, T. N. (1968). Private communication, University of Houston, Houston, Texas. 
LIN, S. H. and Mer, K. K. (1968). Numerical solution of dipole radiation in a compressible plasma. Itt. Trans. Antennas Propagation AP-16, 235.

MELZNER, F. and ROBBEN, H. H. (1970). Ionospheric electron density measurements with improved rocketborne plasma frequency probe. Plasma Waves in Space and in the Laboratory, Vol. 2, pp. 77.77 Edinburgh University Press, Scotland.

MiLler, E. K. (1967). Admittance of the infinite cylindrical antenna in a lossy compressible, anisotropic plasma. Can. J. Phys. 45, 4019.

MiLler, E. K. (1968). Admittance of the infinite cylindrical antenna in a compressible, anisotropic plasma. Can. J. Phys. 46, 1109.

Miller, E. K. and Schulte, H. F. (1970). Antenna admittance in an ionospheric-type plasma. Plasma Waves in Space and in the Laboratory, Vol. 2, pp. 337-370. Edinburgh University Press, Scotland.

NAKATANI, D. T. and Kuehl, H. H. (1973). Input impedance of a short dipode antenna in a warm anisotropic plasma. Submitted to Radio Sci.

Schulte, H. F., Miller, E. K. and Kuiper, J. W. (1968). Ionospheric electron density measurements, Part II. A comparison of experiment and theory for an antenna in the ionosphere. Presented at Spring URSI Meeting, Washington, D.C. 